\section{First report of Wohlfahrtimonas chitiniclastica bacteraemia in South Africa}

To the Editor: The first reported case of Wohlfahrtiimonas chitiniclastica infection in South Africa presented as a soft-tissue infection and the organism was cultured from pus. ${ }^{[1]}$ We describe, to our knowledge, the first case in South Africa of W. chitiniclastica bacteraemia.

The case occurred in a 17-year-old male patient who was admitted to the orthopaedic department of Tygerberg Hospital, Cape Town after sustaining a degloving injury to his right shoulder. He presented with a history of his upper arm being caught in a wood press. The patient lives in a house with running water, electricity and proper ablution facilities. He had no history of excessive alcohol abuse or smoking.

The patient was haemodynamically stable with a degloving injury of his right upper arm and shoulder (Fig. 1). Contamination of the wound with foreign material was minimal. No compartment syndrome was evident. The patient had decreased deltoid and bicep function but distally the wrist and hand were neurovascularly intact. The leucocyte count was $7.93 \times 10^{3}$ cells $/ \mu \mathrm{L}$ and the creatinine level $61 \mu \mathrm{mol} / \mathrm{L}$.

An aerobic blood culture grew W. chitiniclastica. The isolate was identified by matrix-assisted laser desorption ionisation-time of flight mass spectrometry (MALDI-TOF MS). 16S rRNA sequencing confirmed the isolate as $W$. chitiniclastica based on $100 \%$ sequence identity to W. chitiniclastica strain DZ2015 (GenBank: KU301339.1)

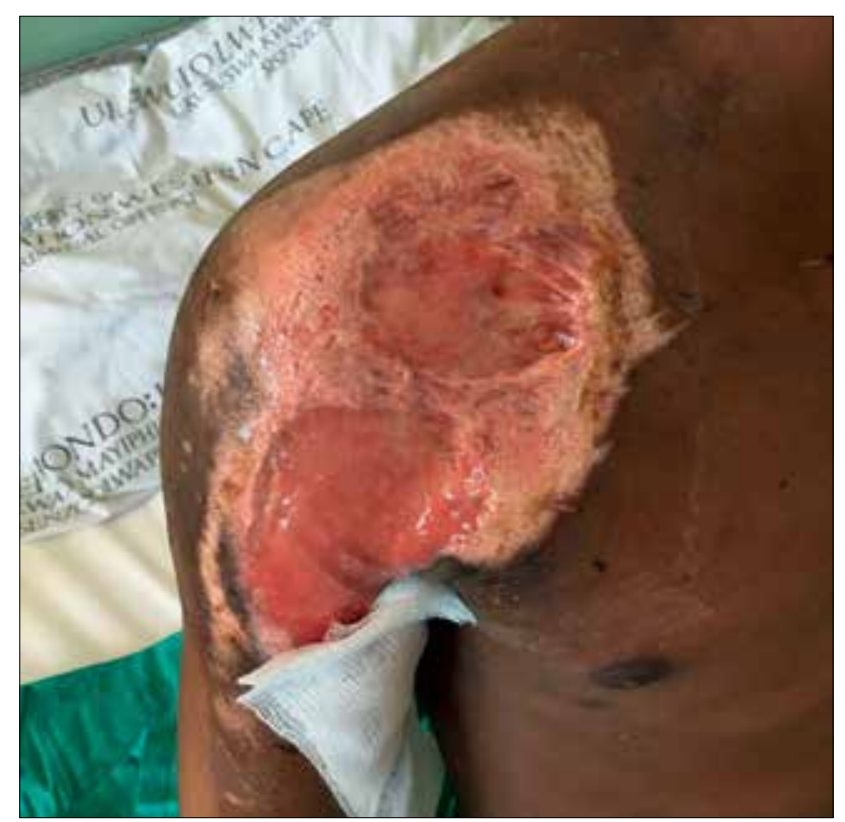

Fig. 1. Right shoulder soft-tissue infection was the most likely source of W. chitiniclastica bacteraemia in this patient. over the 724 bp sequence. ${ }^{[2]}$ Antimicrobial drug susceptibility testing was performed using the Kirby Bauer method and interpreted according to CLSI 2016 criteria for Enterobacteriaciae. The isolate was sensitive to all drugs tested, except for cotrimoxazole, which tested resistant.

The patient was discharged after a course of ceftriaxone $1 \mathrm{~g}$ intravenously daily and successful skin grafts to the affected area. The most likely source of the $W$. chitiniclastica bacteraemia in this patient was the wood-related soft-tissue infection, although maggots were never observed in his wound.

$W$. chitiniclastica is a gram-negative, facultative anaerobic gammaproteobacterium. ${ }^{[3]}$ It was first isolated from the larvae of the Wohlfahrtia magnifica fly. ${ }^{[4]}$ This fly has been reported as the cause of myiasis in live vertebrates in Spain, France, Hungary, Turkey, Egypt, Iran, and Korea. ${ }^{[5]}$

This report should help increase clinicians' awareness of this rare zoonotic pathogen and alert diagnostic microbiology laboratories that the bacteria can currently only be identified using mass spectrometry technology and molecular methods.

Acknowledgement. We gratefully acknowledge the assistance of Dr Mischka Moodley at AmPath Laboratories in identifying the isolate.

\section{R Hoffmann}

Division of Medical Microbiology, Faculty of Medicine and Health Sciences, Stellenbosch University, Tygerberg, Cape Town; and National Health Laboratory Service, Tygerberg Hospital, Cape Town, South Africa renah@sun.ac.za

\section{F Fortuin}

Division of Orthopaedic Surgery, Faculty of Medicine and Health Sciences, Stellenbosch University, Cape Town, South Africa

\section{Newton-Foot, $S$ Singh}

Division of Medical Microbiology, Faculty of Medicine and Health Sciences, Stellenbosch University, Tygerberg, Cape Town; and National Health Laboratory Service, Tygerberg Hospital, Cape Town, South Africa

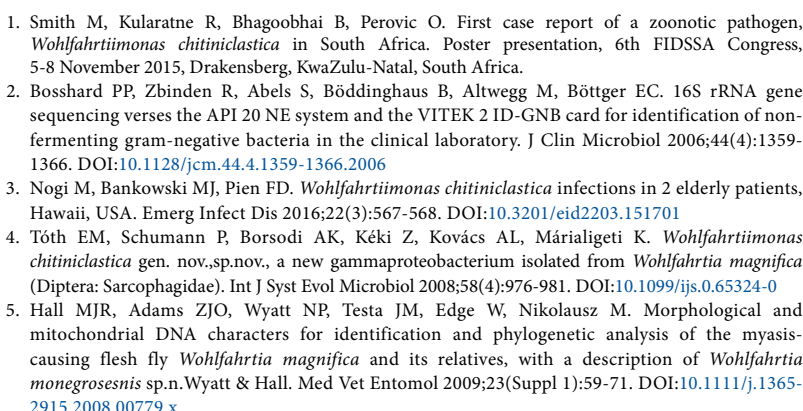
Whifahrtiimonas chitiniclastica in South Africa. Poster presention, 5-8 November 2015, Drakensberg, KwaZulu-Natal, South Africa.

2. Bosshard PP, Zbinden R, Abels S, Böddinghaus B, Altwegg M, Böttger EC. 16S rRNA gen sequencing verses the API $20 \mathrm{NE}$ system and the VITEK 2 ID-GNB card for identification of nonfermenting gram-negative bacteria in the clinical laboratory. J Clin Microbiol 2006;44(4):13591366. DOI: $10.1128 / \mathrm{jcm} \cdot 44.4 .1359-1366.2006$

3. Nogi M, Bankowski MJ, Pien FD. Wohlfahrtiimonas chitiniclastica infections in 2 elderly patients, Hawaii, USA. Emerg Infect Dis 2016;22(3):567-568. DOI:10.3201/eid2203.151701

4. Tóth EM, Schumann P, Borsodi AK, Kéki Z, Kovács AL, Márialigeti K. Wohlfahrtiimonas chitiniclastica gen. nov.,sp.nov, a new gammaproteobacterium isolated from Wohlfahrtia magnifica (Diptera: Sarcophagidae). Int J Syst Evol Microbiol 2008;58(4):976-981. DOI:10.1099/ijs.0.65324-0

5. Hall MJR, Adams ZJO, Wyatt NP, Testa JM, Edge W, Nikolausz M. Morphological and mitochondricical an causing flesh fly Wohlfahrtia magnifica and its retatives with a desciptis of the myasiscausing flsh hy Wohf hins $2915.2008 .00779 . x$

S Afr Med J 2016;106(11):1062. DOI:10.7196/SAMJ.2016.v106i11.11449 